\title{
STATISTICAL PATTERN RECOGNITION FOR STRUCTURAL HEALTH MONITORING USING ESN FEATURE EXTRACTION METHOD
}

\author{
Jianxi Yang,* Gaocen Sha, ${ }^{*}$ Yingxin Zhou, ${ }^{* *}$ Guiping Wang,* and Boren Zheng*
}

\begin{abstract}
For structural dynamic response signal, as the traditional feature extraction methods based on statistical pattern recognition are linear and insensitive, this paper proposes a new signal feature extraction method based on the echo state network (ESN). The proposed method is suitable for analysis of signal with non-linear characteristics. It is more sensitive on the damage identification than the existing methods based on statistical pattern recognition. The proposed method collects structural dynamic response signals under different conditions and uses the ESN network for system identification. The output weights are served as the damage feature values of the bridge structure. A number of experiments are performed by using the non-linear vibration finite element model and a real bridge scale model under environmental motivation. The experimental results show that the proposed non-linear feature extraction method based on ESN is more sensitive than the traditional auto-regressive model. There are obvious differences in the damage sensitivity index value between the healthy case and the damage case. The damage sensitivity index increases linearly with the structural degradation. Moreover, this paper constructs the damage sensitive index by using the Euclidean distance which is consistent with the evolution trend of the structural state. The proposed method offers a more appropriate theoretical and technical support for the existing bridge monitoring data processing and the structural damage evolution trend assessment.
\end{abstract}

\section{Key Words}

Statistical pattern recognition, non-linear feature extraction, ESN, dynamic response signal, damage sensitivity index, bridge monitoring

* College of Information Science and Engineering, Chongqing Jiaotong University, Chongqing 400074, China; e-mail: yjx@cqjtu.edu.cn, \{753710131, 82520252, 9870274\}@qq.com

** Yunnan Communications Investment \& Construction Group Co., Ltd, Yunnan, China; e-mail: zyx6668@126.com Corresponding author: Jianxi Yang

Recommended by Prof. Simon X. Yang

(DOI: 10.2316/Journal.206.2018.6.206-4686)

\section{Introduction}

With the rapid development of pattern recognition, it has been widely applied in the field of structural damage diagnosis. How to find the sensitive characteristics of damage still remains one of the key problems of structural damage identification [1].

Generally, the key step in pattern recognition is feature extraction, which is to find the optimal combination of features as a reflection of the structural damage information. Worden et al. [2] and Sohn et al. [3] use Mahalanobis distance-based outlier detection to realize structural damage identification, where the transmissibility function and the coefficients of the auto-regressive (AR) model are used as damage-sensitive features, respectively. Gul and Catbas [4] use AR model in conjunction with Mahalanobis distance-based outlier detection algorithms to identify different types of structural changes on different test structures. In summary, the signal feature extraction method based on the AR model has been widely used, and the damage features constructed by AR models' coefficients have better recognition properties. However, there are many difficulties to be solved, such as how to determine model orders and how to quantify damage.

Signal feature extraction method based on the AR model is reversible or approximate reversible. Therefore, a signal is corresponding to a unique feature vector. Moreover, less information is lost during the feature extraction process [5]. Therefore, signal feature extraction method based on AR model is showing good performance in both signal classification research and in the field of bridge health monitoring, thus becoming the most commonly used feature extraction methods in bridge structure response signal analysing [6]. However, the modern time-series analysis has been gradually developed into the multi-dimension ARMA (auto-regressive and moving average) model, the non-stationary time-series model, nonlinear multidimensional time-varying time sequence model, and more complex system model, etc. On the other hand, many scholars have not studied the essential characteristics of the signal response obtained from bridge structures. 
Many structural response data from health monitoring show non-linear characteristics because of the impact of external factors. Although the AR model is a kind of linear prediction model, it can only model with the stationary random signal. It cannot accurately describe the significant non-linear characteristics of signals. Enhanced with the non-linear time-series non-stationarity, the model order will be increased as well as the parameter estimation of computational complexity. The calculation accuracy will also be influenced by the length of the sample [7]. The feature extraction method based on the AR model does not have the optimal parameter estimation criterion and model order norms; thus, these models cannot have a better fitting of non-linear time-series.

As non-linear features of the bridge structure response signal are widely realized, a lot of non-linear dynamics index are used for bridge structural response signal feature extraction, such as the maximum Lyapunov exponent, fractal dimension, and approximate entropy. However, the calculation process of the non-linear dynamics index is complex, time-consuming. In addition, many indexes in the calculation process are not able to get the exact value.

To solve the aforementioned bottlenecks of damage feature extraction methods, this paper proposes a novel bridge monitoring signal feature extraction method based on the theory of echo state network (ESN). ESN is a new type of recurrent neural network which can describe chaotic time series well. To date, ESN has been widely applied in the field of non-linear system modelling because of its dual advantage in forecasting accuracy and efficiency. For example, Dominey et al. [8] establish mammalian brain signal sequence model by using ESN mechanism. Hinton and Salakhutdinov [9] achieve a non-linear dimension reduction of image data with the help of ESN non-linear processing ability. Han and Sun [5] carry out the research of automatic detection and classification on the electroencephalography signal and present a novel feature extraction method based on ESN. Miao [10] carries out a study of speech recognition method based on ESNs; Wang [11] studies non-linear time-series forecasting by the ESN modelling.

For the bridge structure system, due to the structure itself and the non-linear materials, coupled with the effects of random factors in the external environment, bridges and other structural systems exhibit complex non-linear dynamic behaviour. Numerous structural response data from the health monitoring system are bound to exhibit non-linear characteristics [12]. Therefore, methods based on ESN can be applied for feature extraction of bridge monitoring signal, thus revealing the mechanism of signal non-linear evolution. Because bridge structural response signal is a dynamic time series, a new type of feature extraction method for bridge structural response signal can be obtained by using ESN instead of the AR model. This method has a strong non-linear processing capacity and has all the advantages of the AR model. In addition, the training process is fast and not easy to fall into local optima because it is only needed to adjust the output weights and the efficiency is higher. After introduced the savings pool, the training process of ESN solely works on the input set to change the output connection weight matrix. It greatly reduces the computation and complexity and avoids the slow convergence and local optima (commonly existing in gradient descent algorithm), thus obtaining excellent modelling accuracy.

\section{Statistical Pattern Recognition for Structural Health Monitoring Based on ESN Feature Extraction Method}

\subsection{Bridge Structural Response Signal Feature Extraction Method Based on ESN}

In the field of statistical pattern recognition, feature extraction is defined as extracting representative active ingredients from an existing pattern vector (initial characteristics) and constituting a new feature vector (fine features) [13], [14], which can reduce workload and improve efficiency and accuracy, as well as provide the foundation for further statistical discrimination [15].

ESN network is characterized by its core structure, which is a randomly generated and remains unchanged "Reservoir". ESN is large-scale and sparse-connected recursive neural network; wherein, the input weights, the internal connection weights, and output feedback weights are randomly generated, and once generated, they are no longer changed. In ESN, the output weight is the only part which needs to be adjusted. Simple linear regression can be used to train ESN network. The main idea of ESN is to use the input signal to excite state response signal of the reserve pool; then, a linear combination of the state response signal is used to approximate target vector [16].

The ESN state equation is given by the following:

$$
\begin{gathered}
x(n+1)=f\left(W x(n)+W_{\text {in }} u(n)+W_{\text {back }} y(n)\right) \\
\hat{y}(n+1)=f_{\text {out }}\left(W_{\text {out }}[x(n+1), u(n+1), y(n)]+W_{\text {bias }}^{\text {out }}\right)
\end{gathered}
$$

where $W, W_{\text {in }}$ and $W_{\text {back }}$ are state variables, the connection weight matrix of input and output state variables, respectively. $W_{\text {out }}$ is the output connection weight matrix. $\quad x(n+1), u(n+1), y(n)$ record the combined motivation vector; $W_{\text {bias }}^{\text {out }}$ is the output bias or noise. $f=f\left[f_{1}, f_{2} \ldots, f_{N}\right]$ is the internal neuron activation function. $f_{i}(i=1,2, \ldots, N)$ is the hyperbolic tangent function. $f_{\text {out }}=\left[f_{\text {out }}^{1}, f_{\text {out }}^{2}, \ldots, f_{\text {out }}^{L}\right]$ is output function. Typically, $f_{\text {out }}^{i}(i=1,2, \ldots, L)$ is an identity function. In the training of the network, the connection weight matrices $W, W_{\text {in }}$, and $W_{\text {back }}$ which connected to the reservoir are generated randomly, and once generated, they are no longer changing, but the output connection weight matrix $W_{\text {out }}$ must be obtained by training. The output connection weight matrix $W_{\text {out }}$ is calculated as following:

$$
\begin{aligned}
& \left(W_{\text {out }}\right)^{t}=M^{-1} T \\
& W_{\text {out }}=\left(\left(W_{\text {out }}\right)^{t}\right)^{t}
\end{aligned}
$$


The steps of bridge monitoring signal feature extraction method based on the ESN are as follows:

(1) The first step is to initialize the network, first of all, determine the parameters of input node number $K$, output node number $L$, and the size $N$ of the reservoir, then randomly generated the input connection weight matrix $W_{\text {in }}$ of (1), internal connection weight matrix $W$, and feedback connection weight matrix $W_{\text {back }}$.

(2) The second step is training, input $W_{\text {in }}, W$, and $W_{\text {back, }}$, the training data is sequentially input to the reservoir and generate incentives according to (1), then stored the reservoir state $x(n)$ in the matrix $M$, the vector $\mathrm{T}$ consisting of the output values corresponding to the input training data, finally, according to (3) and (4) to calculate the output connection weight matrix $W_{\text {out }}$.

(3) The third step is testing, output the test vector and obtained the internal excitation state according to (1), then you can get the output in accordance with (2).

(4) Finally, the reservoir network are used to identify each block of bridge structural response signals, the output value of ESN model for each segment of the bridge monitoring signals served as characteristics.

\subsection{Structural Health Monitoring Based on Statis- tical Pattern Recognition}

Based on the statistical pattern recognition theory, after the completion of structural damage detection system modelling and feature extraction, there is a need to establish the discriminant function that based on extracted features for classification to distinguish the health case and abnormal case. This paper is used ESN for bridge structure response signal features extraction and constructed damage sensitivity index based on multivariate statistical methods, then distinguished the evolution trend of the structure state by the distribution of the damage index. The steps are as follows:

(1) Data acquisition and pre-processing: Collected bridge monitoring data $X(t)$ under different structural states, the data obtained from structure under the healthy case for each measuring point as a reference sample $\mathrm{R}$, the data obtained from healthy to damage case for each measuring point as test samples $\mathrm{T}$, standardized the each sample data:

$$
x(t)=\frac{X(t)-\mu}{\sigma}
$$

where $\mu$ is the mean value of $X(t)$ and $\sigma$ is the variance.

(2) Parameter selection and feature extraction: Cutting the time-series data which obtained from each measuring point into the same uniform blocks according to the collection frequency, sets up ESN model for all the sample data to obtain the output weights. First, select the ESN network status dimension $\mathrm{N}$, internal network connection weights $W_{\text {in }}$, sparsity $c$ and the spectral radius $r$, and randomly generated a dynamic network reservoir according to the above parameters; then set the initial state of the network, and according to the network state evolution equation (2) excited the state $x(n)$ of ESN network by the input signal $u(n)$; finally, the output weights is calculated by the state matrix $W$ of the ESN network and the target vector $y$ and served as damage feature vector of bridge monitoring signal.

(3) Statistics discriminant: The $k$ feature vectors were obtained by above steps, the distance of Euclidean between each test sample feature vector and reference sample feature vector is calculated, defined the distance as damage sensitivity index $D_{d s i}$ :

$$
D_{d s i}=\sum_{i=1}^{n} \sqrt{\sum_{j=1}^{m}\left(x_{k j}-y_{i j}\right)^{2}} \quad(k=1,2, \ldots, n)
$$

where $x_{k j}$ is the Euclidean distance between $k$ th damage feature vector of each measuring point and reference sample, $y_{i j}$ is reference sample.

(4) Damage discrimination: Discriminant the evolution trend of the structure state by the distribution of the damage index.

\section{Experimental Study}

\subsection{The Simulation Test Model}

In this paper, MIDAS CIVIL software was used to establish the pier simulation model (Fig. 1), the height is $10 \mathrm{~m}$ and the section is $1.5 \times 1.5 \mathrm{~m}^{2}$, using three linear model of skeleton curve (Fig. 2) to establish a non-linear structural vibration simulation model based on non-elastic hinge. The damage occurred because of the structure into the plastic, and bridge has entered the non-linear working range, only for structural non-linear time history response analysis can realistically simulate the actual structural response.

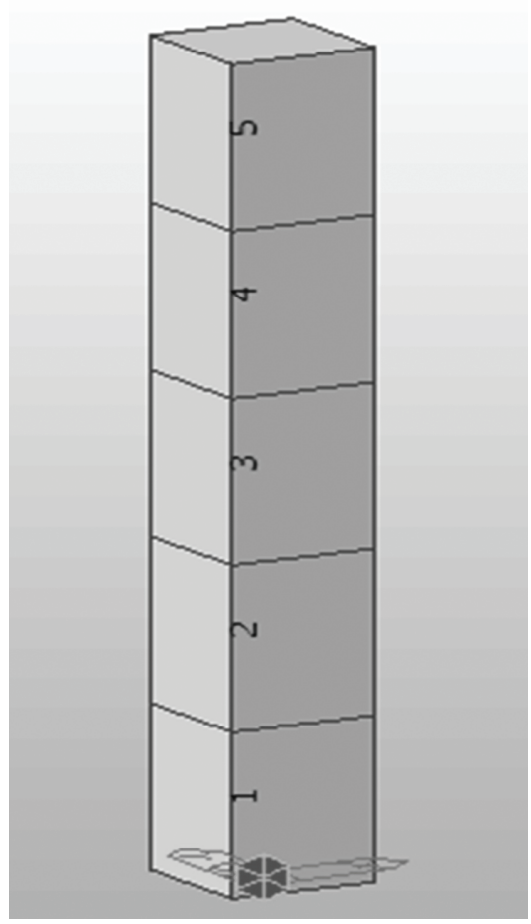

Figure 1. A bridge pier simulation model. 


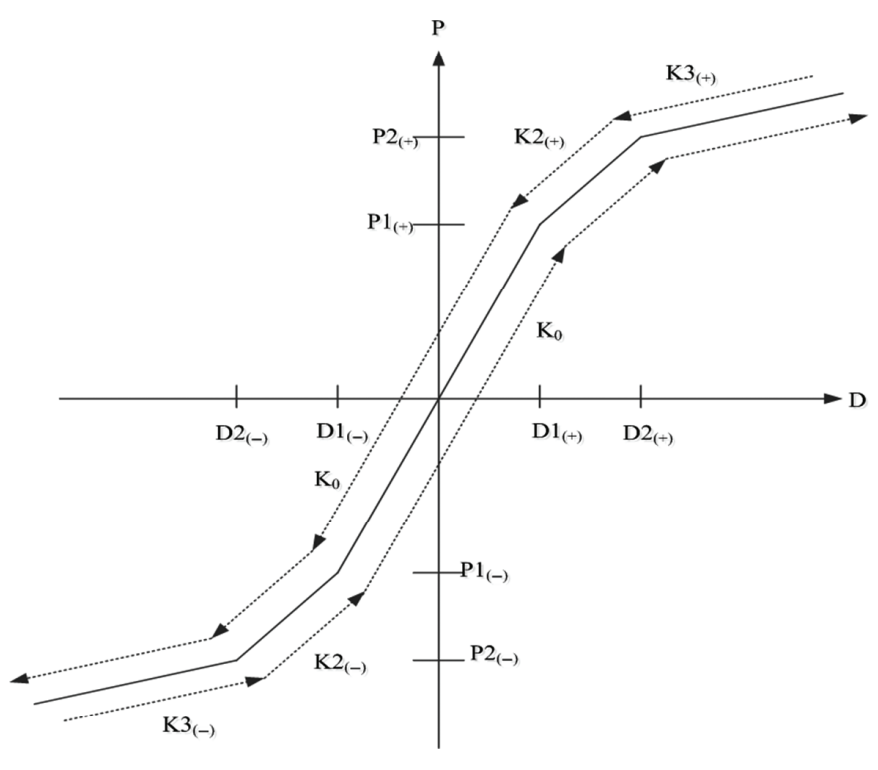

Figure 2. Skeleton curve of three linear model.

Therefore, this paper uses the plastic hinge to analysis bridge dynamic elastic-plastic, the non-linear displacement ductility ratio was served as the criteria to discriminant the damage degree to the structure (where is the maximum displacement of non-linear response, is the structural yield displacement). By adding the seismic load resulting in varying degrees of structural damage, the load condition is: 0.001 times of org-wv2, 0.003 times of org-wv2, 0.006 times of org-wv2, 0.01 times of org-wv2. The ductility ratio under four conditions is obtained by FEM calculation, this paper adopted ductility ratio as evaluation index of structural damage, the units number of ductility ratio which is greater than 1 served as a measure of structure damage degree.

Thus, it can be divided into following situation depending on the calculation result:

(1) The load is 0.001 times of org-wv2, structural ductility ratio is less than 1 , it is served as a structural healthy case.

(2) The load is 0.003 times of org-wv2, the ductility ratio of Units 1 and 2 are greater than 1, in this situation, it is served as damage Case 1.

(3) The load is 0.006 times of org-wv2, the ductility ratio of Units 1,2, and 3 are greater than 1, in this situation, it is served as damage Case 2 .

(4) The load is 0.01 times of org-wv2, the ductility ratio of all the units are greater than 1 except for Unit 5 , in this situation, it is served as damage Case 3 .

Finally, obtained pier non-linear dynamic structural acceleration signal of $2-6$ measuring point under different conditions by MIDAS CIVIL software.

\subsection{Experimental Analysis}

The acceleration data obtained from 2, 3, 4, 5, 6 units are used for experiment, the pre-10s data obtained from healthy case for each unit served as reference sample $R$ and the $10 \mathrm{~s}-20$ s data obtained from healthy to damage case for each unit served as test samples T. Cutting the time-series data which obtained from each measuring point into the same uniform blocks according to the collection frequency, sets up ESN model for training, this paper uses a standard ESN with 96 internal nodes, namely $n$ is 96 , the spectral radius $\alpha$ of the internal connection weight matrix is $0.8, W_{\text {bias }}^{\text {out }}$ is 0.0001. Obtaining output weights and served as damage feature vector of bridge monitoring signal, constructed damage feature vector matrix $G=\left[G_{1}, G_{2}, \ldots, G_{16}\right]$ for each measuring point under different conditions, calculated the Euclidean distance between each feature vector $G_{i}$ and the reference sample, obtained the distribution of damage sensitivity index under different conditions, as shown in Fig. 3.

Comparing Figs. 3 and 4, it is apparent that the bridge monitoring information feature extraction method based on the ESN has obvious superiority than traditional AR model for non-linear data processing, and the damage index constructed on the basis above has a strong sensitivity for the evolution of the structural state. The evolution of structural state can be assessed by changes of the damage sensitivity index. The experiment results show that this bridge structural response signal feature extraction method shows a good performance, this method can realize the non-linear characteristic extraction of the bridge structural response signal, and the feature extraction process is approximately reversible with less information loss. In this paper, the proposed feature extraction method in the process of bridge response signal feature extracting, mainly concentrated in the state solution of the pseudo-inverse matrix, so the computational efficiency is higher than the traditional time series method.

\subsection{Bridge Scale Model Experiment}

The methodology is further elucidated by taking an experiment of the Lab existing 1:20 scale model of Heichong Gou Bridge (Figs. 5 and 6).

The test structure obtained data by using 13 piezoelectric accelerometers, the accelerometers are placed in structural roof top surface, the outer webs, the bottom surface of $1 / 8 \mathrm{~L}, 3 / 16 \mathrm{~L}, 1 / 4 \mathrm{~L}, 3 / 8 \mathrm{~L}, 1 / 2 \mathrm{~L}$ main span (the location can be seen in Fig. 7).

Various damage conditions are simulated by artificial kerf. Healthy case with no cracks in midspan as non-destructive condition, damage Case 1 with $0.1 \mathrm{~mm}$ cracks in midspan; damage Case 2 with two cracks $(0.12 \mathrm{~mm}+0.03 \mathrm{~mm})$ in midspan; damage Case 3 with two cracks $(0.12 \mathrm{~mm}+0.07 \mathrm{~mm})$ in midspan.

The test is excited by random excitation with a sampling frequency of $18.8 \mathrm{~Hz}$, the data were collected for $50 \mathrm{~s}$ after the exciter works for $5 \mathrm{~s}$. For the representative measuring Point 1, Point 7, Point 10, Point 13, and Point 17, the first 10s data under healthy case are used as reference sample and the data ranges from 10 s to 20 s are used as test sample, the data block is constructed by 10 times the cycle of data, as a result, each point has 12 data blocks. Establishing ESN model for each data block and obtained the output weights to build damage feature vectors, the calculated Euclidean distance between the test sample and 


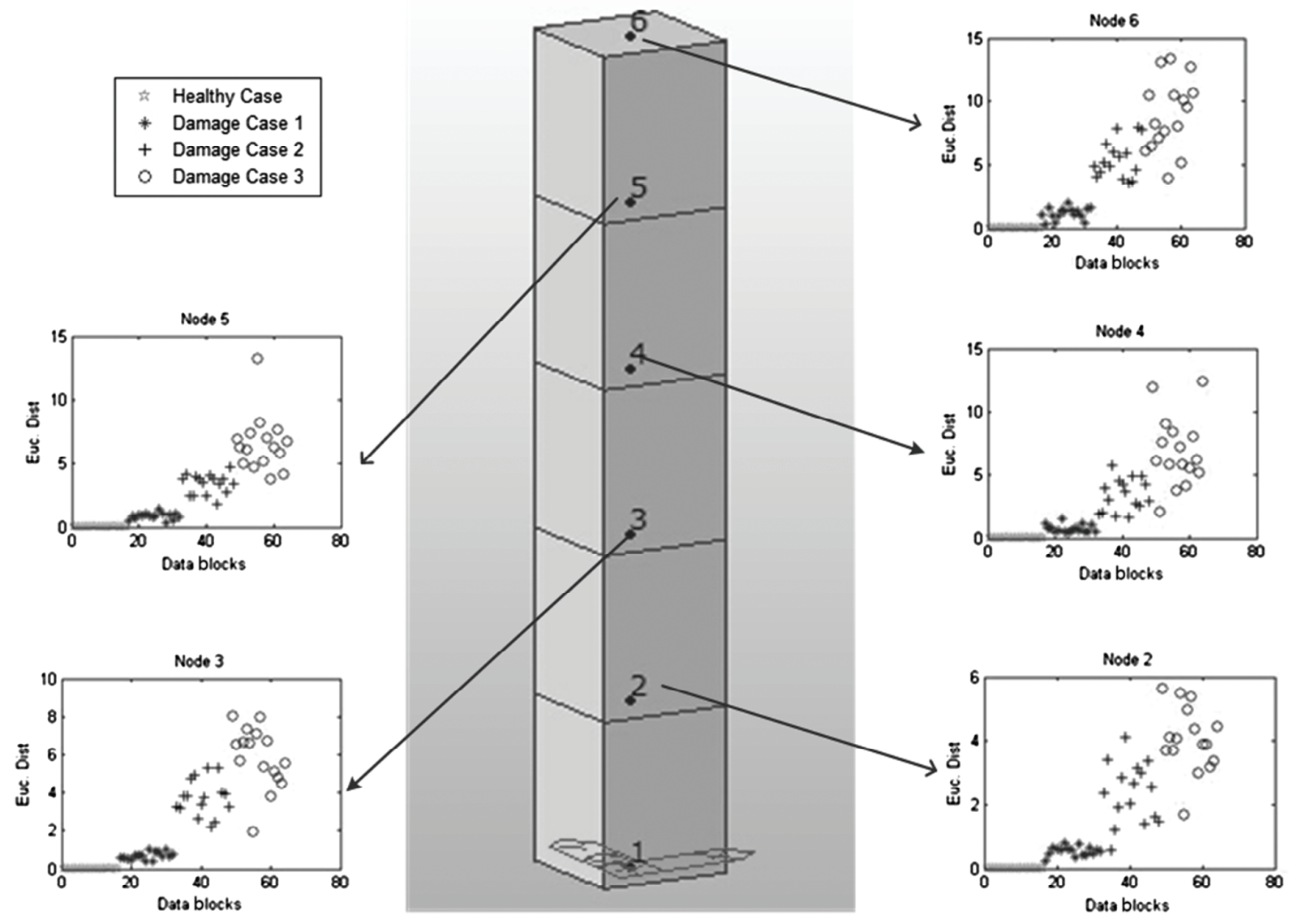

Figure 3. The distribution of damage sensitive indicators $D_{d s i}$ based on ESN feature extraction methods.
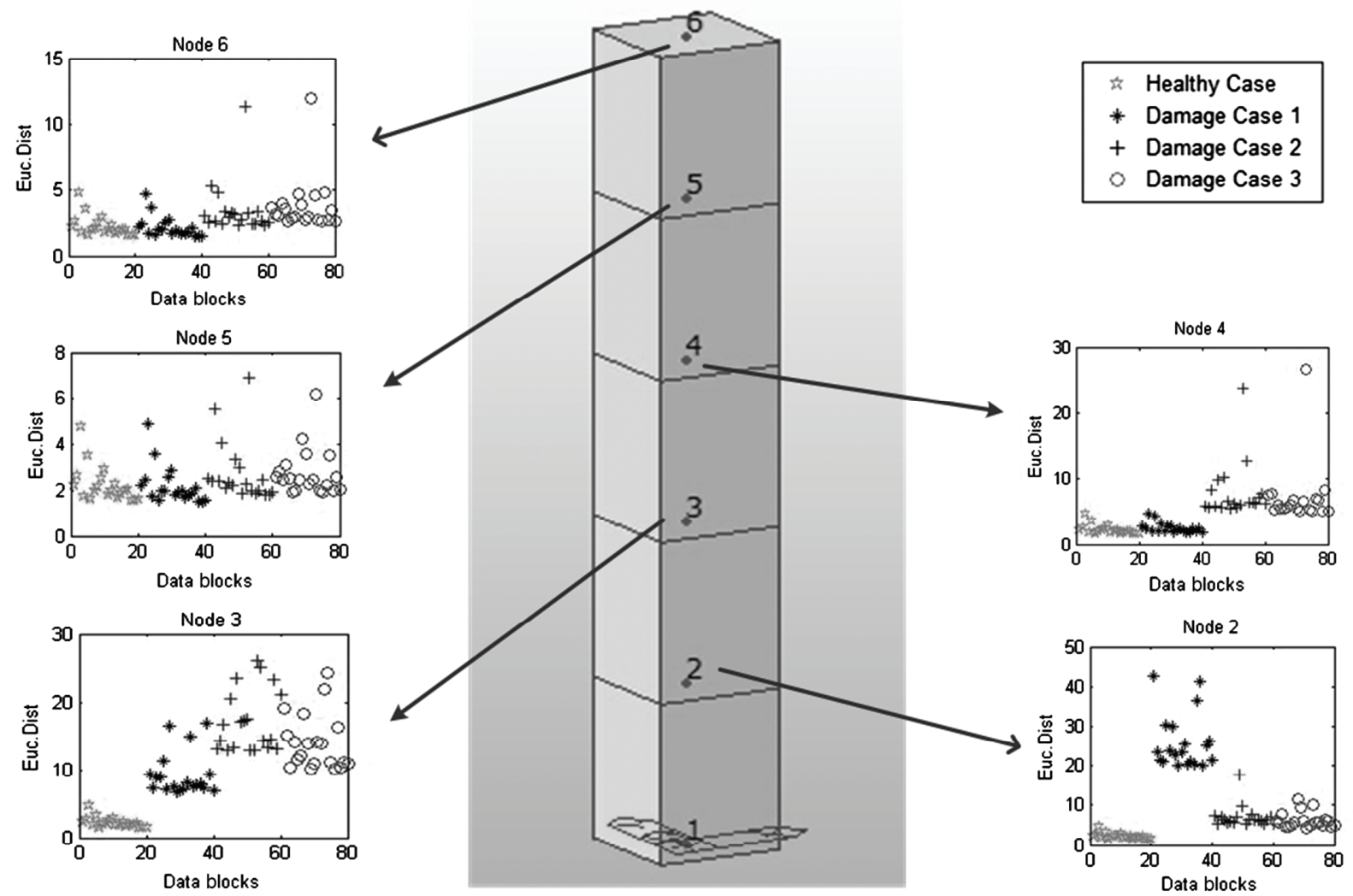

Figure 4. The distribution of damage sensitive indicators $D_{d s i}$ based on AR feature extraction methods. 


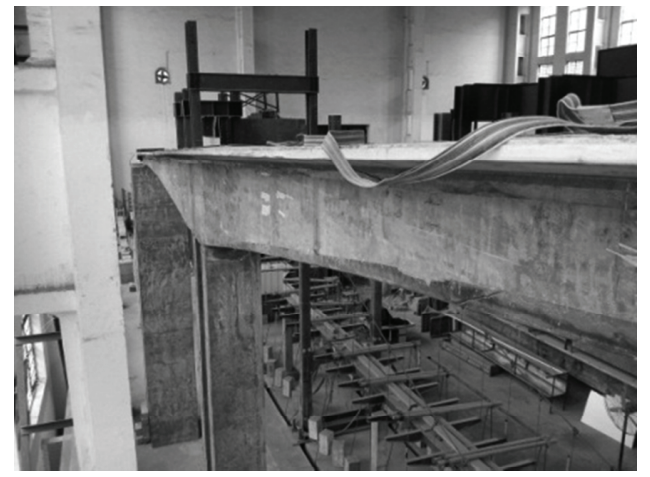

Figure 5. Reduced scale model of bridge structure. the reference sample is served as damage sensitivity index $D_{d s i}$, Fig. 8 shows the plots of $D_{d s i}$ with abovementioned points under different case.

As shown in Fig. 8, the real bridge structural response signals are seriously influenced by the random factors of the external environment, as well as the bridges and other structural systems exhibit complex nonlinear dynamic behavior. Moreover, numerous structural response data obtained from the health monitoring system are bound to exhibit nonlinear characteristics, and then the damage features obtained by echo state network trained data can effectively characterize the evolutionary trends of the bridge structural state. Therefore, it is proved that the feature extraction method has a strong sensitivity for structural damage detection.

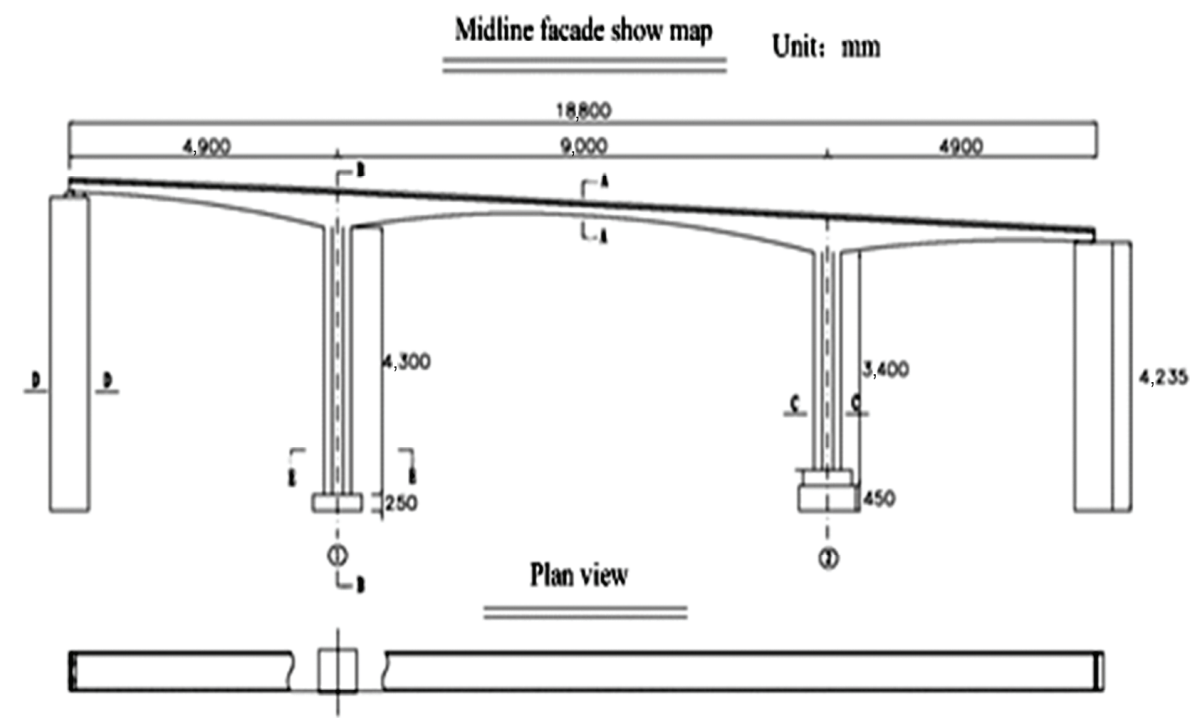

Figure 6. Overall layout of the mode.

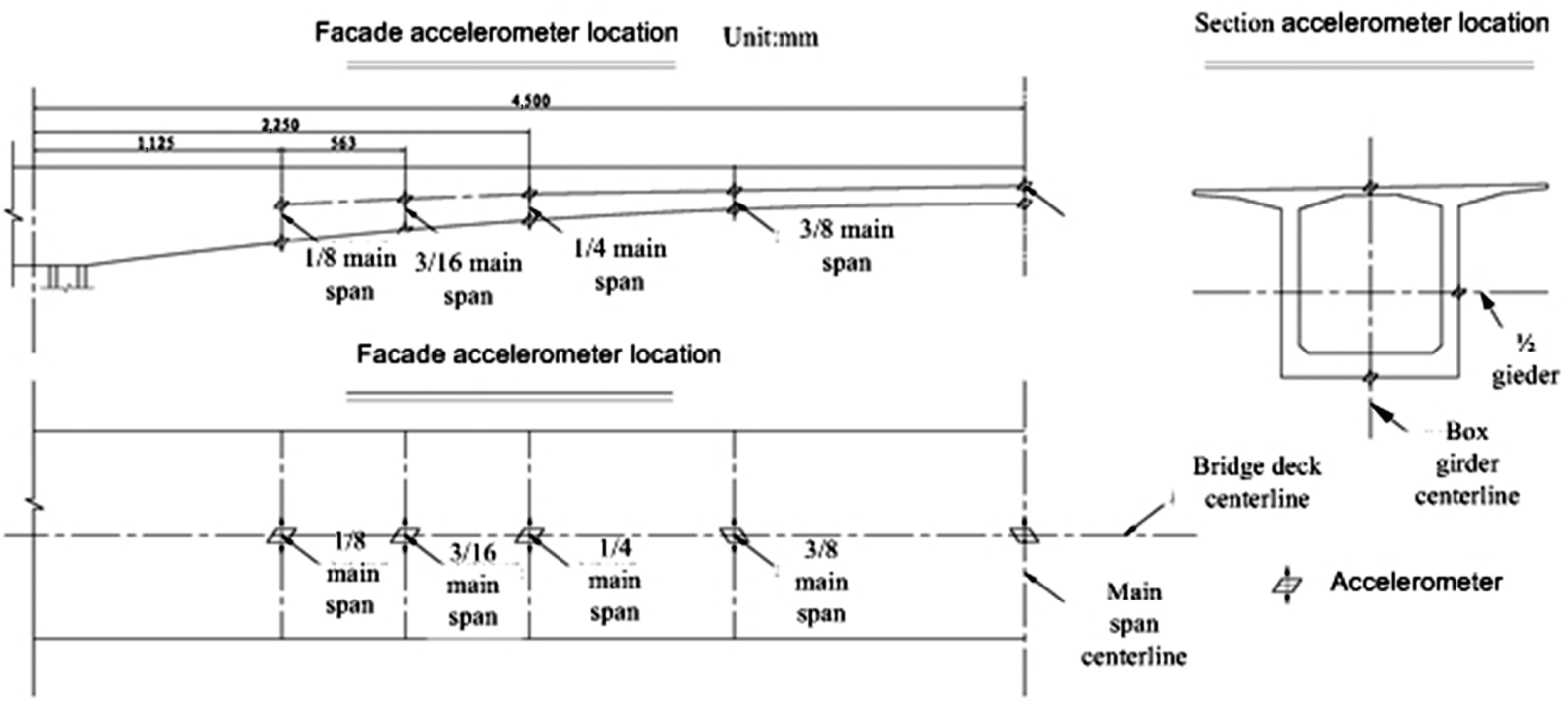

Figure 7. Accelerometer location. 

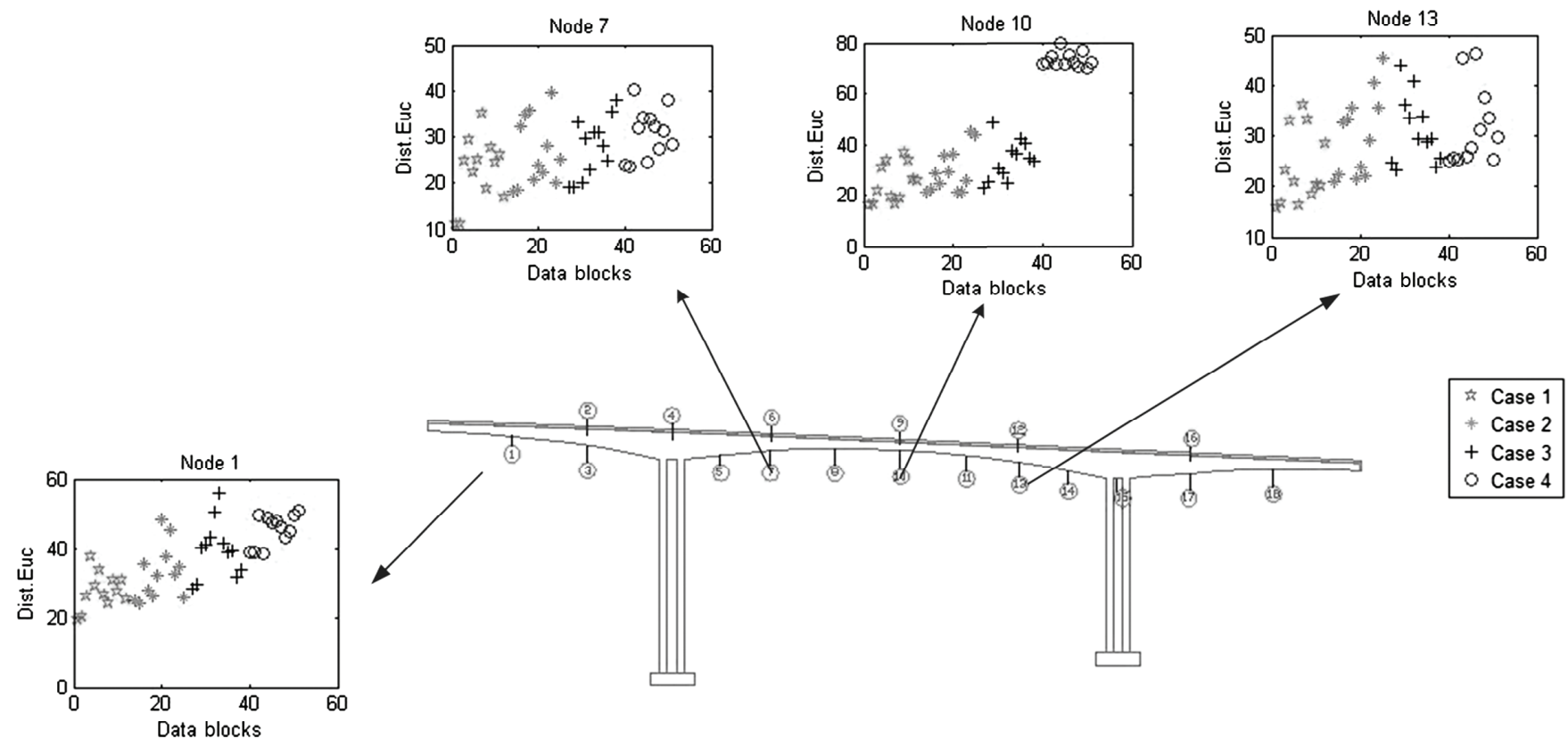

Figure 8. The distribution of damage sensitive indicators based on ESN model feature extraction methods.

\section{Conclusion}

This paper analyses the advantages and disadvantages of the traditional feature extraction methods for bridge structure response signal under the framework of statistical pattern recognition. It proposes a new signal feature extraction method based on non-linear dynamics theory, i.e., a feature extraction method for bridge structural response signal based on the ESN. It analyses the non-linear characteristics of the non-linear dynamic response for bridge acceleration time series signal (the signal must contains the structural non-linear dynamic response information) by the ESN model. It reveals the signal non-linear evolution mechanism which characterized the structural damage degradation from the perspective of signal non-linear characteristic.

This paper uses the plastic hinge for the elastic-plastic dynamic analysis. The non-linear displacement ductility ratio is served as the criteria to discriminant the damage degree to the structure. It collects bridge structural response signals in different conditions, adopts the damage feature extraction method based on the AR model and the ESN model respectively, and gets the distribution of damage sensitive indicators of different measuring point under various conditions. In the traditional feature extraction method based on the AR model, each measurement point has no significant change in the distribution of damage sensitive indicators under different conditions. While the results obtained by the ESN model present a linear growth trend as the bridge structural response, signal shows non-linear characteristics. Therefore, the ESN model for non-linear feature extraction is better than traditional AR model, and the magnitude of each data is $10^{-6}$. The results of the damage index based on the Euclidean distance can effectively characterize the evolution of the structural state and the sensitivity is very high.
Through practical data verification, the effectiveness of the proposed method is further verified. It is concluded that the feature extraction method based on ESN is sensitive to damage. It improves the stability of the methods for structural damage identification.

\section{Acknowledgement}

This work of this paper is supported by National Natural Science Foundation (Grant No. 51208538 and 51278512), Science and Technology Innovation Talents of Chongqing (Grant No. CSTCKJCXLJRC17), Chongqing Research Program of Basic Research and Frontier Technology (Grant No. cstc2015jcyjBX0014, cstc2016jcyjA0304, cstc2016jcyja0022), Yunnan science and technology plan project (Grant No. 2017IB025).

\section{References}

[1] Y.L. Chang and J.J. Leou, Representation and matching of feature patterns for robot operation monitoring, International Journal of Robotics 85 Automation, 10(4), 1995, 143-151.

[2] K. Worden, G. Manson, and N.R.J. Fieller, Damage detection using outlier analysis, Journal of Sound and Vibration, 229(3), 2000, 647-667.

[3] H. Sohn, C.R. Farrar, N.F. Hunter, and K. Worden, Structural health monitoring using statistical pattern recognition techniques, Journal of Dynamic Systems, Measurement, and Control, 123(4), 2001, 706-777.

[4] M. Gul and F.N. Catbas, Statistical pattern recognition for structural health monitoring using time series modeling: Theory and experimental verifications, Mechanical Systems and Signal Processing, 23(7), 2009, 2192-2204.

[5] M. Han and L.L. Sun, Extraction of EEG feature extraction based on echo state networks, Journal of Biomedical Engineering, 29(2), 2012, 207-208.

[6] X. Wang, K. Smith, and R. Hyndman, Characteristic-based clustering for time series data, Data Mining and Knowledge Discovery, 13(3), 2006, 335-364. 
[7] X.M. Tao, J. Xu, L.B. Yang, and Y. Liu, Bearing fault diagnosis with a MSVM based on a GARCH model, Journal of Vibration and Shock, 29(5), 2010, 11-15.

[8] P.F. Dominey, M. Hoenm, and T. Inui, A neurolinguistics model of grammatical construction processing, Journal of Cognitive Neuroscience, 18(12), 2006, 2088-2107.

[9] G.E. Hinton and R.R. Salakhutdinov, Reducing the dimensionality of data with neural networks, Science, 313(5786), 2006, 504-507.

[10] J. Miao, The research of speaker-independent isolated word speech recognition method based on the echo state network (Gansu: Lanzhou University, 2012).

[11] J.M. Wang, Nonlinear time series prediction method based on echo state network (Hei Longjiang: Harbin Institute of Technology University, 2011)

[12] J.X. Yang, J.T. Zhou, and F.Q. Han, Study on state recognition of ASCE benchmark FEM based on Lyapunov exponent spectrum entropy, Applied Mechanics and Materials, 117-119, 2012, 1526-1527.

[13] X.D. Li, C.M. Luo, J. Dezert, and Y.Z. Tan. Generic object recognition based on feature fusion in robot perception, International Journal of Robotics and Automation, 31(5), 2016, 409-415.

[14] C. Ulas and H. Temeltas, Feature-based 3d outdoor slam with local filters, International Journal of Robotics and Automation, 28(3), 2013, 226-233.

[15] Y. Liu and Y.G. Li, Structural damage diagnosis method and application research based on statistical pattern recognition (Nanjing: Southeast University, 2009).

[16] H. Jaeger, Echo state network, Scholarpedia, 2007, http://www. scholarpedia.org/article/Echo_state_network.

\section{Biographies}

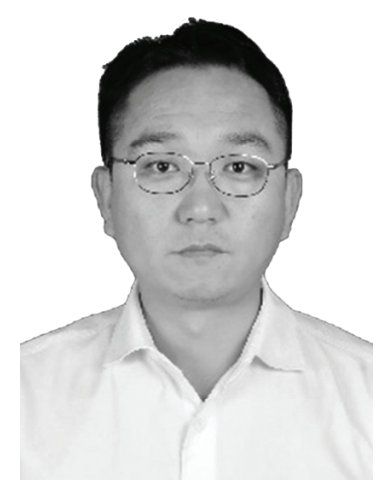

Jianxi Yang received his B.S. degree in Chongqing Jiaotong University, P.R. China, at 2000; received his M.S. degree in Chongqing University, P.R. China, at 2007; and received his Ph.D. degree at Chongqing Jiaotong University, P.R. China, in 2011. Currently he is a professor in College of Information Science and Engineering, Chongqing Jiaotong University, P.R. China. His research interests include structural control \& health monitoring, structure security evolution.

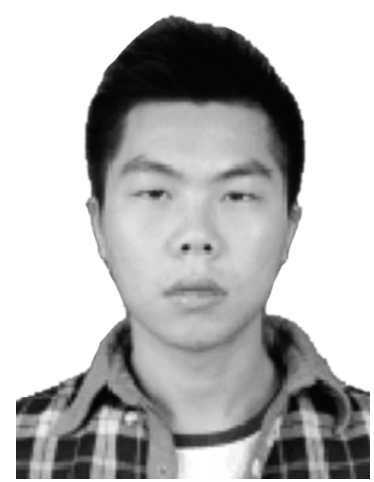

Gaocen Sha is a graduate student in Chongqing Jiaotong University.

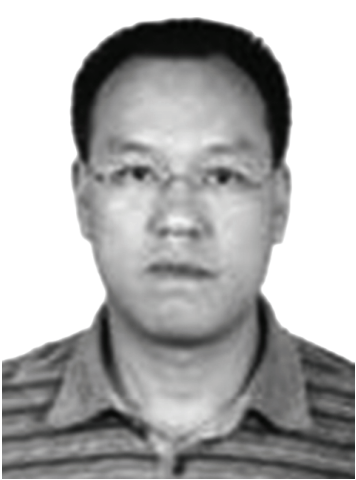

Yingxin Zhou is a senior engineer in Yunnan Communications Investment \& Construction Group Co., Ltd, P.R. China. He mainly engages in the design and construction of bridges and roads.

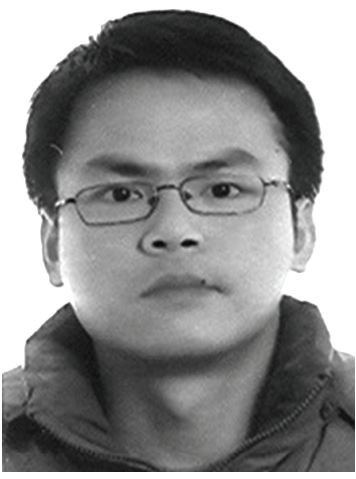

Guiping Wang received his B.S. degree, M.S. degree, and Ph.D. degree at Chongqing University, P.R. China, in 2000, 2003, and 2015, respectively. Currently he is an associate professor in College of Information Science and Engineering, Chongqing Jiaotong University. His research interests include machine learning, dependability analysis, and design of systems. As the first author, he has published overs 20 papers in related research areas during recent years.

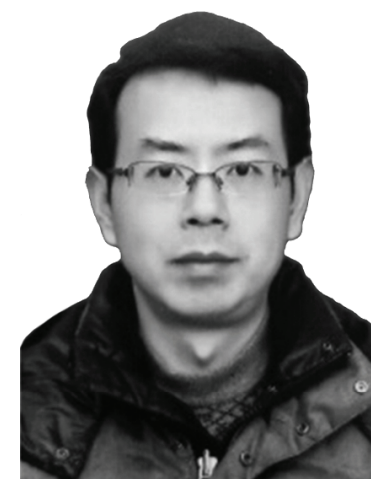

Boren Zheng received the B.Sc. degree in electronic information engineering from Southwest University, China in 1997, the M.Sc. degree in electronic circuit and system, and the Ph.D. degree in microwave technology from University of Electronic Science and Technology of China, in 2005 and 2012, respectively. His research interests include RF\&MW circuit and system, electronic technology in the bridge health monitoring, prestress detection in structure, and the technology of directional sound. 